\section{BMJ Open} Ophthalmology

\title{
Acupuncture and herbal formulation compared with artificial tears alone: evaluation of dry eye symptoms and associated tests in randomised clinical trial
}

\author{
Louis Tong, ${ }^{1,2,3,4}$ Hla Myint Htoon, ${ }^{1,3}$ Aihua Hou, ${ }^{1,3}$ Rajendra Udyavara Acharya, ${ }^{5,6,7}$ \\ Jen-Hong Tan, Qi-Ping Wei, ${ }^{8}$ Pat Lim ${ }^{9}$
}

To cite: Tong L, Htoon HM, Hou A, et al. Acupuncture and herbal formulation compared with artificial tears alone: evaluation of dry eye symptoms and associated tests in randomised clinical trial. BMJ Open Ophthalmology 2018;3:e000150. doi:10.1136/ bmjophth-2018-000150

- Additional material is published online only. To view please visit the journal online (http://dx.doi.org/10.1136/ bmjophth-2018-000150).

Received 18 January 2018 Revised 11 May 2018 Accepted 11 May 2018

A) Check for updates

${ }^{1}$ Singapore Eye Research Institute, Singapore

${ }^{2}$ Singapore National Eye Centre, Singapore

${ }^{3}$ Duke-NUS Graduate Medical School, Singapore

${ }^{4}$ Yong Loo Lin School of

Medicine, National University of Singapore, Singapore

${ }^{5}$ Ngee Ann Polytechnic, Singapore

${ }^{6}$ Department of Biomedical Engineering, SIM University, Singapore

${ }^{7}$ Department of Biomedical Engineering, University of Malaya, Kuala Lumpur, Malaysia ${ }^{8}$ Beijing University of Chinese Medicine, Beijing, China ${ }^{9}$ Singapore Chung Hwa Medical Institution, Singapore

Correspondence to Dr Louis Tong; louis.tong.h.t@ singhealth.com.sg

\section{ABSTRACT}

Objective Dry eye is a common disease with great health burden and no satisfactory treatment. Traditional Chinese medicine, an increasingly popular form of complementary medicine, has been used to treat dry eye but studies have been inconclusive. To address this issue, we conducted a randomised investigator-masked study which included the robust assessment of disease mechanisms.

Methods and analysis Eligible participants (total 150) were treated with artificial tear (AT) alone, with added eight sessions of acupuncture (AC) or additional daily oral herb (HB) over a month.

Results Participants treated with AC were more likely to respond symptomatically than those on AT ( $88 \%$ vs $72 \%, p=0.039)$ with a difference of $16 \%(95 \% \mathrm{Cl}: 0.18$ to 31.1). The number-to-treat with $A C$ to achieve response in one person was 7 ( 3 to 157). Participants in the AC group also had reduced conjunctival redness (automatic grading with Oculus keratograph) compared with AT ( $p=0.043)$ and reduced tear T helper cell (Th1)-cytokine tumour necrosis factor $\alpha(p=0.027)$ and Th2-cytokine interleukin 4 concentrations $(p=0.038)$. AC was not significantly superior to AT in other outcomes such as tear osmolarity, tear evaporation rates, corneal staining and tear break-up times. No significant adverse effects were encountered. HB was not significantly different in the primary outcome from AT $(80 \%$ vs $72 \%, p=0.26)$.

Conclusions $\mathrm{AC}$ is safe and provides additional benefit in mild to moderate dry eye up to 1 month, compared with ATs alone. Treatment is associated with demonstrable molecular evidence of reduced inflammation. Provided that suitably qualified practitioners are available to implement standardised treatment, AC may be recommended as adjunctive therapy to AT.

Trial registration number ClinicalTrials.gov (NCT02219204)registered on 14 August 2014.

\section{INTRODUCTION}

Dry eye is a highly prevalent multifactorial disease of the tear and ocular surface, affecting $14 \%$ to $33 \%$ of the adult population worldwide and is more common in Asia. Mild to moderate forms of dry eye are even more common and associated with significant healthcare and economic burden worldwide. It is a holistic problem affected by living habits,

\section{Key messages}

What is already known about this subject?

Dry eye can result from diminished tear production or tear instability, and though very common, treatment is unsatisfactory. Traditional Chinese medicine has been used in dry eye, but studies reported conflicting results.

\section{What are the new findings?}

- Here, we found in a randomised comparative trial that adjunctive use of acupuncture reduced the symptoms of dry eye in participants, induced a reduction of conjunctival redness and decreased tear concentration of tumour necrosis factor $\alpha$, suggesting that acupuncture has an anti-inflammatory effect on the ocular surface compared with artificial tears.

\section{How might these results change the focus of research or clinical practice? \\ > This study suggests that acupuncture treatment may be another option in the treatment of mild to moderate dry eye and more studies should focus on whether acupuncture reduces inflammation.}

nutrition and underlying systemic disease, inducing a significant decrease in quality of life. ${ }^{12}$

Dry eye can result from diminished tear production, but tear instability usually plays a role. Despite the heterogeneous nature of dry eye aetiologies, common disease hallmarks are raised tear osmolarity and inflammation. Current treatments available are not satisfactory; they include tear supplements, anti-inflammatory medication, oral supplements and tear retention methods, for example, punctal occlusion and moisture chamber eyewear. In the majority of dry eye sufferers, who have mild to moderate disease, the management is with topical lubricants and control of aggravating factors. ${ }^{2}$

From the traditional Chinese medicine (TCM) perspective, systemic yin-deficiency 
refers to a syndrome where yin chi (energy) in the body is too weak to counterbalance yang chi, which results in malfunction of a typical set of body organs, since all organs require the correct balance of energies for harmony or proper physiological function. When yin-deficiency syndrome is encountered, the earliest manifestation of imbalance would be fluid deficiency, and dry eye symptoms may emerge from this deficiency. TCM encompasses various treatment modalities, most commonly acupuncture (AC) and herbal (HB) medications. A recent meta-analysis concluded that AC improved dry eye signs such as tear break-up time, Schirmer I test result and corneal fluorescein dye staining. ${ }^{3}$ Nevertheless, randomised controlled studies evaluating $\mathrm{AC}$ and $\mathrm{HBs}$ in dry eye conducted previously included various bias and produced mixed results. ${ }^{4-10}$ Previous TCM trials in dry eye did not classify the patients according to TCM syndromes, which is not desirable. ${ }^{11}$ Previous studies also lacked objective assessment for inflammation, tear evaporation or tear osmolarity, which forms the scientific basis of dry eye. One study used objective imaging and showed that tear meniscal parameters increased 4 weeks after AC, but mechanistic explanation was not provided. ${ }^{12}$ With increasing popularity of TCM, there is significant urgency to scientifically evaluate it.

We aimed to assess the effectiveness of $\mathrm{AC}$ and a $\mathrm{HB}$ formulation in dry eye in a randomised comparative clinical study, with the inclusion of objective and robust outcomes including tear osmolarity, tear evaporation rates and inflammatory markers.

\section{MATERIALS AND METHODS}

Study design

This was a randomised investigator-masked parallel group interventional trial.The history of amendments is provided in the website https://clinicaltrials.gov/ct2/ show/NCT02219204. These study arms were involved: (1) A common comparative group with only Systane Ultra (Alcon, Fortworth, Texas, USA) eye-drop (artificial tear (AT) $)(\mathrm{n}=50),(2)$ Systane Ultra with AC $(\mathrm{n}=50)$ and (3) Systane Ultra and HB treatment $(n=50)$. The duration from baseline to follow-up assessment was 4 weeks. All participants were screened and recruited within 1 year. The study adhered to Consolidated Standards of Reporting Trials guidelines.

\section{Participants}

Written informed consent was obtained from participants at the Singapore Chung Hwa Medical Institution (SCHMI). This is an institute staffed mainly by volunteer healthcare workers and physicians but accepts private consultation fees for outpatient appointments.

For inclusion, participants satisfied each of the following: age of 40-85 years; visual acuity better than Snellen acuity of 6/60; chief complaint was dry eye; dry eye symptom (SPEED) score $>6$; TCM score $(0-33)$ of $>6$ for lung-kidney yin deficiency; based on interviewer-administered questionnaire, a higher score indicates more yin deficiency (online supplementary efigure 1); signs of dry eye (tear break-up time $<10 \mathrm{~s}$ or Schirmer's test $<10 \mathrm{~mm} / 5$ $\mathrm{min})$; and any corneal fluorescein dye staining. Adults older than 40 years were included in the study as the HB formulation, one of the treatments to be evaluated, was customised for older adults.

Participants with any of the following criteria were excluded: (1) ophthalmic disease, for example, extraocular muscle palsies, ectropion, entropion, trichiasis, glaucoma, ocular allergies, for example, allergic conjunctivitis, sinusitis, eczema, atopic keratoconjuntivitis, eye surgeries including LASIK (laser-assisted in situ keratomileusis, within 1 year); eye-related trauma or contact lens wear (within 1 year) or punctal occlusion, (2) requirement for ciclosporin and steroidal eye-drops during the study period, (3) systemic disease requiring regular medication (except hypertension and lipidaemia), for example, physician-diagnosed thyroid disorders, StevensJohnson syndrome, Sjogren's syndrome, (4) requirement for medications such as antimicrobial, inflammatory, creams (except moisturisers or cosmetics), (5) pregnancy or (6) TCM treatment within past month. The usage of AT prior to the study is provided in online supplementary etable 1 . All these eye-drops have been stopped in the trial period.

\section{Randomisation and masking}

Randomisation was performed at SCHMI, where members were unmasked as they delivered TCM treatments. The randomisation was done using SPSS V.17 software with a witness present using the randomisation process at the commencement of the study. A random number 1,2 or 3 was allocated to an integer between 1 and 150 corresponding to each participant to be recruited, in such a way that there would be 50 participants in each group. The code was then kept by an administrative staff not involved in the assessment of the participants. Study information related to randomisation were filed and kept at SCHMI and only unmasked personnel could access the codes. Singapore Eye Research Institute personnel, except a single study coordinator, were designated to evaluate the outcome measures and thus were masked to prevent biases.

\section{Overall hypothesis}

The overall hypothesis is that TCM modalities of AC and HBs increase the proportion of people with improvement in dry eye symptoms above those using only Systane Ultra eye-drops; these TCM treatments also improve the clinical signs of dry eye, and objectively, the TCM-induced effects should be linked to mechanism of dry eye: reduction of tear evaporation, tear osmolarity or reduction of inflammatory markers such as tear cytokines. In addition, the ocular effects should also be associated to the expected treatment-induced change in the TCM systemic syndrome. 


\section{Outcomes}

The primary outcome was the proportion of participants who achieved any improvement in symptom (SPEED) score at week 4 compared with the baseline (symptom responder). This proportion was compared between the AC and AT groups, and between the HB and AT groups.

These secondary efficacy outcomes were assessed at week 4 and baseline visits and differences were computed: tear evaporation rates, non-invasive tear break-up time (NIBUT), Schirmer I, corneal fluorescein staining (five zones), tear osmolarity, tear cytokine levels and TCM score (lung-kidney yin-deficiency). The differences were then compared between AC vs AT and HB vs AT groups.

Proteins were eluted using our previous protocol. ${ }^{13}$ The Schirmer strips were longitudinally halved, and from the half strip, tear cytokines were evaluated using the multiplex bead-based indirect immunofluorescent assay (Milliplex; Merck-Millipore, Billerica, Massachusetts, USA). Cytokine concentrations were normalised to the length of wetting of the Schirmer strips $+3 \mathrm{~mm}$. The advantage of this technique was that 15 cytokines could be assayed using a minimum amount of tear.

Potential adverse events of $\mathrm{HB}$ consumption were monitored: nausea, insomnia, headache, loss of appetite, malaise, diarrhoea and abdominal cramps. Side effects after AC that were monitored include soreness, bruises, fatigue, lightheadedness and involuntary twitching of muscles. Intraocular pressures were measured using non-contact tonometry, and presenting visual acuity was measured with the LogMAR (logarithm of the minimum angle of resolution) charts.

\section{Study treatment}

All participants used Systane ultra (Alcon) eye-drops four times a day. AC patients undergone twice weekly AC over eight sessions, and HB patients used 'qi ju gan lu yin' twice daily. (Details of treatments are provided in online esupplementary materials, etable 2 and supplementary efigure 2).

\section{Statistical analysis}

Sample size calculation was based on the main clinical outcome (proportion of responders), and a two sample test of proportions power analysis was conducted with Sample Size calculator software: http://powerand samplesize.com/Calculators/Compare-2-Proportions.

To achieve a significance level $(\alpha)$ of 0.05 and a power of $80 \%$, assuming proportion of responders to be 0.7 in $\mathrm{AT}$ and 0.9 in $\mathrm{AC}$ (or $\mathrm{HB}$ ), that is, $20 \%$ more responders in the TCM group and a sampling ratio of 1,47 participants were required in each of the groups. To allow for $5 \%$ lost-to-follow up, 50 participants were recruited per group.

Analysis was performed after all subjects had completed required visits or withdrawn from the study. The analysis was by intention-to-treat, applied on the right eye for eye-specific data. For the primary outcome, the proportions of responders were assessed as hypothesised above, the $\mathrm{X}^{2}$ test was used to evaluate statistical significance at the one-tailed $\mathrm{p}<0.05$. The confidence limits of the difference in proportions were determined using the Wilson's method.

For secondary outcomes, independent sample t-tests were performed comparing the changes in the AC or $\mathrm{HB}$ group with the AT group, with statistical significance set at the $\alpha$ level of 0.05 using the Holm-Sidak method (GraphPad Prism 6 software; GraphPad, San Diego, California, USA). A one-tailed test was used according to the hypothesis tested, for example, for known proinflammatory cytokine interleukin (IL)-1 $\beta$, the hypothesis was that AC reduced IL-1 $\beta$ more than AT. For a cytokine with known anti-inflammatory role, that is, IL-10, the hypothesis was that AC increased IL-10 more than AT. The Mann-Whitney test was additionally performed for variables that were not normally distributed.

\section{RESULTS}

\section{Participant characteristics}

From March 2015 to December 2015, 179 patients had been screened (figure 1) and 150 participants were recruited (table 1 ), with 116 women and 34 men (mean age $58.3 \pm 7.8$ years). There were no significant differences in any baseline parameter. The participants, apart from having symptoms and staining, only need to have either abnormal Schirmer or NIBUT, and relatively normal results of these two tests suggest that the participants had only mild to moderate dry eye. There was no clear difference in the use of previous lubricating eye-drops in the three groups (online supplementary etable 1).

All patients except one (from HB) completed the study and had follow-up data. There was no difference in the frequency of use of topical eye-drops between the three groups in the study period (data not shown). First, the symptoms (SPEED scores) in each of the three treatment groups had improved significantly $(\mathrm{p}<0.001)$ from baseline (mean $\pm \mathrm{SD}$ changes of $-4.4 \pm 3.7$ in $\mathrm{HB},-4.5 \pm 3.8$ in $\mathrm{AC}$ and $-4.1 \pm 4.1$ in $\mathrm{AT})$.

Figure 2A shows there was a significantly higher proportion of participants experiencing symptomatic improvement in AC $(88 \%, 44 / 50)$ compared with AT $(72 \%, 36 / 50) \quad\left(X^{2}=3.06\right.$, Fisher's exact test $\left.\mathrm{p}=0.039\right)$. This difference was $16 \%$ (95\% CI: 0.18 to 31.1$)$ and the number- to-treat ${ }^{14}$ with AC to achieve response (over and above treatment with AT) was 7 (95\% CI: 3 to 157).

In terms of actual magnitude of improvement, more participants with AC than AT improved 3 to 6 points (figure 2B), the median (IQR) change in the symptom was $-4(-2.75$ to -8.25$)$ and $-3(0$ to -6$)$ in AC and AT, respectively ( $U$ test $\mathrm{p}=0.06$ ). There was no significant difference in the change in SPEED scores between groups although there was a trend for AC to have greater improvement than AT (figure 2B, raw data in online supplementary efigure 3). The difference observed was only with respect to the amount of change of SPEED between the groups but not in the final SPEED scores between groups at 1 month (online supplementary 


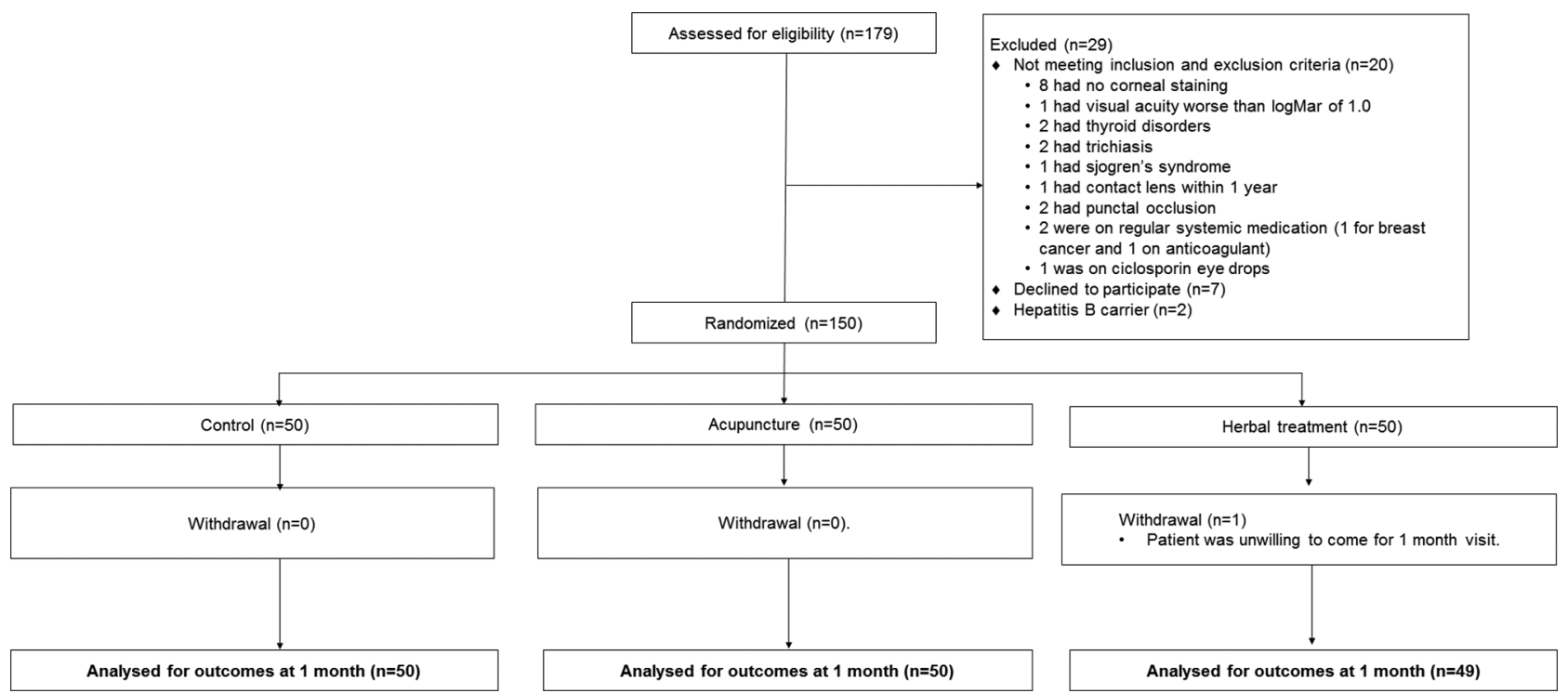

Figure 1 Study flow chart, according to the Consolidated Standards of Reporting Trials. The diagram shows eligibility screening from enrolment to follow-up visit for the determination of study outcomes.

etable 3). In terms of symptom improvement, HB was not significantly different from AT $(80 \%, 39 / 49$ vs $72 \%$, $36 / 50$, respectively, $\mathrm{p}=0.26$ ).

TCM scores measured before and after treatment are shown in online supplementary etable 4 , where a higher score indicates a more severe lung-kidney yin deficiency. Symptom responders and non-responders had similar scores at baseline, but responders had lower post-treatment scores and a significantly larger reduction after treatment.

The secondary outcome measurements at the 4-week follow-up visit are shown in online supplementary etable
3. There was no significant difference between groups, except that AT had higher NIBUT $(9.8 \pm 6.3 \mathrm{~s}$ vs $6.9 \pm 5.1 \mathrm{~s})$ compared with AC $(p=0.01$, U test: $p=0.0092)$. However, when analysing the change in NIBUT between week 4 and baseline, all groups had similar changes: $0.13 \pm 6.29 \mathrm{~s}$ (HB), $-0.66 \pm 5.83 \mathrm{~s}(\mathrm{AC})$ and $0.43 \pm 6.89 \mathrm{~s}(\mathrm{AT})(\mathrm{p}>0.05)$.

Interestingly, there was a significant reduction in the change in conjunctival redness measurement (figure 3 ) in the AC $(-0.052 \pm 0.038)$ compared with AT $(+0.034 \pm 0.031)$ $(\mathrm{p}=0.043)$. As redness was not normally distributed, this analysis was repeated with the non-parametric $\mathrm{U}$ test $(\mathrm{p}=0.038)$. The changes in all other clinical parameters

Table 1 Baseline characteristics of the intention-to-treat population

\begin{tabular}{lllll}
\hline & & Herbs & Acupuncture & Artificial tears \\
\hline Number & & 50 & 50 & 50 \\
\hline Female & $\%$ & 82 & 74 & 76 \\
& Proportion & $41 / 50$ & $37 / 50$ & $38 / 50$ \\
\hline Age & Mean \pm SD & $57.36 \pm 6.45$ & $58.92 \pm 9.17$ & $58.94 \pm 7.92$ \\
\hline SPEED & Mean \pm SD & $11.44 \pm 4.28$ & $12.14 \pm 4.69$ & $11.16 \pm 4.94$ \\
\hline NIBUT $(s)$ & Mean \pm SD & $8.02 \pm 6.13$ & $7.58 \pm 5.44$ & $9.36 \pm 6.34$ \\
\hline Schirmer I $(\mathrm{mm})$ & Mean \pm SD & $13.1 \pm 10.8$ & $10.9 \pm 7.8$ & $11.7 \pm 8.6$ \\
\hline Corneal staining & & & & \\
\hline Central & Mean \pm SD & $0.12 \pm 0.39$ & $0.13 \pm 0.56$ & $0.09 \pm 0.31$ \\
\hline Inferior & Mean $\pm S D$ & $0.9 \pm 0.9$ & $1.05 \pm 1.03$ & $0.87 \pm 0.86$ \\
\hline Superior & Mean $\pm S D$ & $0.37 \pm 0.5$ & $0.46 \pm 0.6$ & $0.36 \pm 0.58$ \\
\hline Temporal & Mean $\pm S D$ & $0.21 \pm 0.47$ & $0.54 \pm 0.87$ & $0.33 \pm 0.61$ \\
\hline Nasal & Mean $\pm S D$ & $0.3 \pm 0.67$ & $0.57 \pm 0.97$ & $0.39 \pm 0.39$ \\
Tear osmolarity (mOsm) & Mean $\pm S D$ & $300 \pm 12.2$ & $301 \pm 12.8$ & $305 \pm 16$ \\
Conjunctival redness & Mean $\pm S D$ & $1.19 \pm 0.41$ & $1.15 \pm 0.40$ & $1.23 \pm 0.37$ \\
\hline
\end{tabular}

*Standard patient evaluation of eye dryness: greater number (0-28) indicates more severe/frequent dry eye symptoms.

NIBUT, non-invasive tear break-up time. 


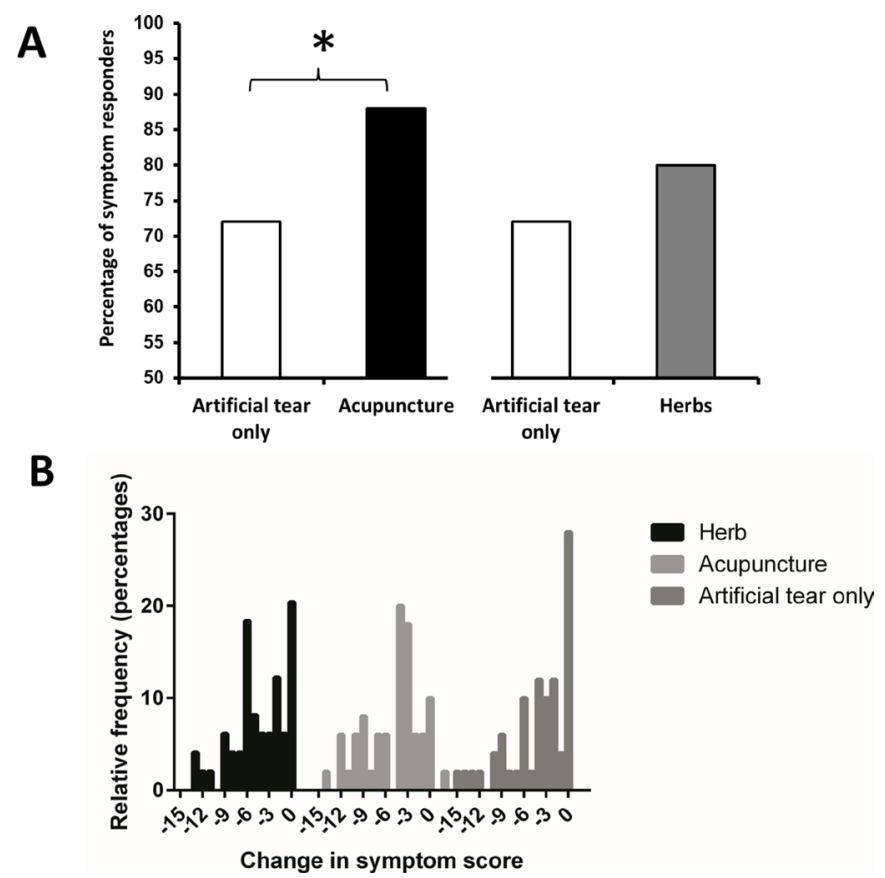

Figure 2 Primary outcome. (A) Proportion of dry eye symptom responders, ${ }^{*} \mathrm{P}=0.039$. (B) Histograms showing change in magnitude of symptoms at week 4 from baseline.

(corneal fluorescein staining, NIBUT, Schirmer I) in AC and $\mathrm{HB}$ were similar in AT (all $\mathrm{p}>0.05$ ).

One patient from $\mathrm{HB}$ and one from AC refused to have osmolarity assessment at week 4 . The tear osmolarity was similar and generally not very high in all groups at baseline (table 1) and at week 4 (online supplementary etable 3 ). There was no significant difference in the change in osmolarities between groups or induced by treatment within each group (online supplementary efigure $4 \mathrm{~A}$ ). In a subset analysis where only participants above 306 mOsm were included, the HB group had a lower osmolarity at the end of study compared with the AC group ( $\mathrm{p}=0.048)$. On the other hand, the inferior cornea staining was slightly worse in the AC group

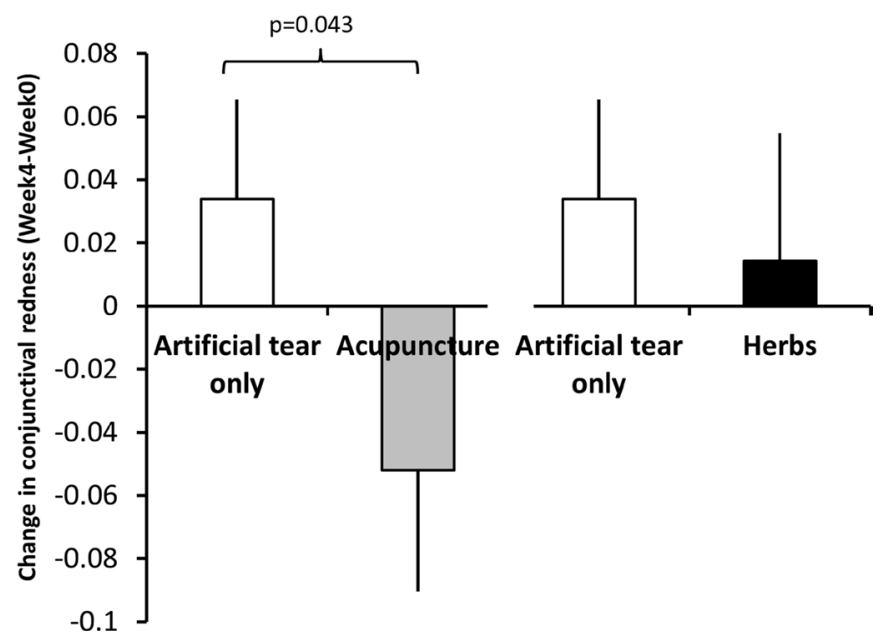

Figure 3 Changes in conjunctival redness after treatment. Height of bars represents mean, and error bars represent SD. compared with the HB group $(\mathrm{p}=0.015)$ and also worse in the AC group compared with AT group $(\mathrm{p}=0.002)$ (online supplementary etable 5 ). Tear evaporation rates (online supplementary efigure 4B) over the conjunctiva or cornea did not change significantly from baseline (all $\mathrm{p}>0.05$ ). These magnitudes were similar to measurements in other patients with dry eye. ${ }^{15}$ Similar findings were obtained when the total area of the conjunctiva and cornea was analysed (data not shown).

The concentrations of tear cytokines at baseline and 4 weeks are shown in online supplementary etable 6 and etable 7), respectively, and actual changes in online supplementary efigure 5 . At the commencement of the study, there was a lower tear concentration of (monocyte chemoattractant protein-1) MCP-1 in HB compared with AT ( $\mathrm{p}=0.006$, using $\mathrm{U}$ test: $\mathrm{p}=0.015)$. AC had higher tear IL-4 compared with AT ( $p=0.025)$, but this was not statistically significant with the $\mathrm{U}$ test $(\mathrm{p}=0.24)$.

The proinflammatory cytokines with differential changes after treatment are shown in figure 4. AC shows a greater reduction $(p=0.027)$ in tear tumour necrosis factor $\alpha$ (TNF- $\alpha)$ (T helper (Th) 1 cytokine) concentrations compared with AT (figure 4) ( $\mathrm{p}=0.039$ on U test). The actual change of the mean TNF- $\alpha$ concentration was from $0.045 \mathrm{pg} / \mathrm{mL}$ to $0.021 \mathrm{pg} / \mathrm{mL}$ in the AC group (online supplementary etables 6 and 7 ). There was no significant change in levels of IL-1 $\beta$ (online supplementary efigure 6). AC also had a greater reduction $(\mathrm{p}=0.038)$ in the tear IL-4 concentrations compared with the AT group (figure 4) ( $\mathrm{p}=0.098$ on $\mathrm{U}$ test). On the other hand, the HB treatment reduced the concentration of tear cytokine IL-12 p70 compared with the AT group (figure 4$)(\mathrm{p}=0.025$, $\mathrm{U}$ test: $\mathrm{p}=0.017)$. The changes of concentrations of all other cytokines did not show a significant difference between groups (all $\mathrm{p}>0.05$ ). If cytokine concentrations were lower in the follow-up examination than the baseline, they were considered as downregulated. We performed an additional analysis of tear cytokines and showed the number of participants with downregulated cytokines in online supplementary etable 8. $\chi^{2}$ tests showed that more participants in $\mathrm{HB}$ than AT group had downregulation of IL-10, and more participants in AC compared with AT had downregulation of IL-1 $\beta$. If we had considered the effect of multiple testing, the $\mathrm{p}$ value that would be significant would be $0.05 / 15=0.0033$, since there were 15 cytokines. Based on this adjusted $p$ value, none of the cytokine concentrations were significantly different between groups. Nevertheless, the actual $p$ values should be noted because the study was not powered for comparing cytokine concentrations, and the sample sizes may be too low to detect significant differences. The participants in the AT group had a significant reduction of (interferon-inducible protein 10) IP-10 ( $\mathrm{p}=0.021)$ but not those in the other two groups (online supplementary efigure 6 ).

Adverse effects were not encountered. The single participant who did not complete follow-up cited social reasons for withdrawal. 
A

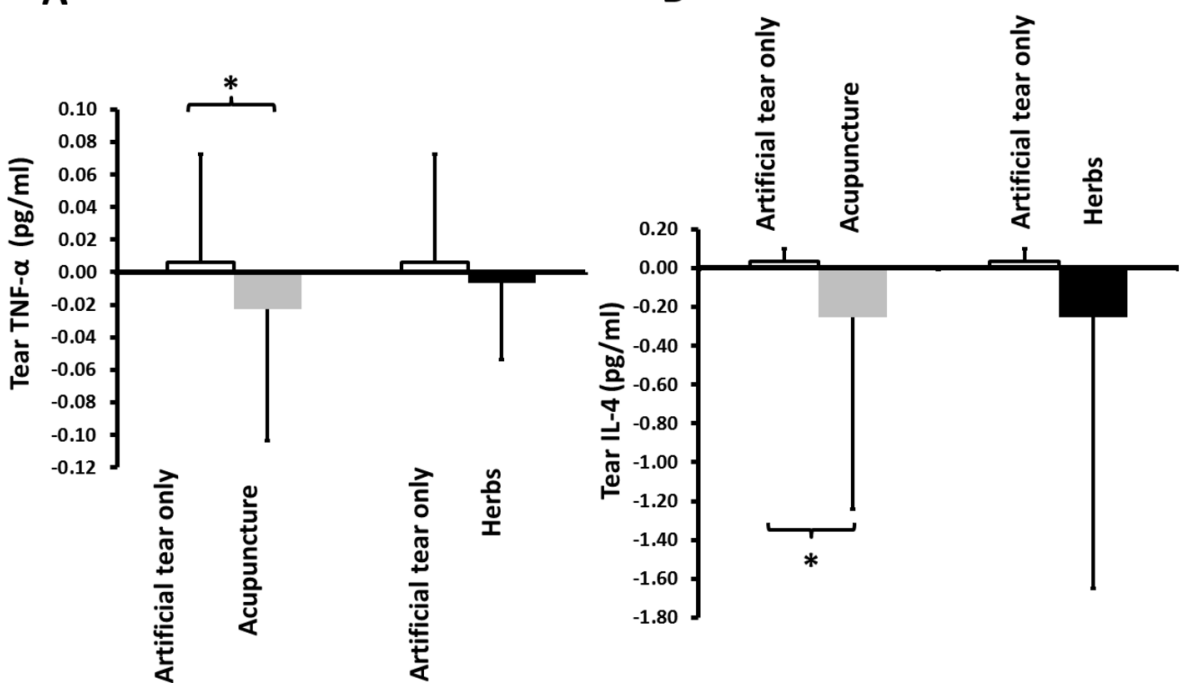

C

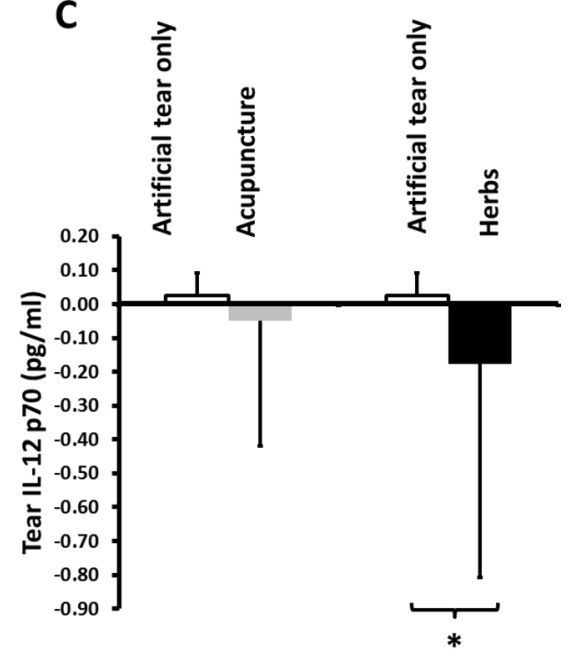

Figure 4 Change in tear cytokine levels after treatment (subtracting week 4 from baseline). (A) Tear tumour necrosis factor $\alpha$ (TNF- $\alpha$ ) concentrations, (B) tear interleukin (IL)-4 concentrations and (C) tear IL-12 concentrations. Height of bars represents mean, and error bars represent SD. ${ }^{*} \mathrm{P}<0.05$.

\section{DISCUSSION}

There have been reported clinical trials on the efficacy of TCM ( $\mathrm{AC}^{7816}$ and HB medication), ${ }^{5}$ including two systematic analyses and a meta-analysis. ${ }^{3}{ }^{310}$ In contrast with our study, Shin et al showed that tear break-up time can be improved by AC, ${ }^{717}$ whereas Chang et $a \tilde{l}$ showed that tear break-up time and Rose Bengal staining were improved by HBs. ${ }^{5}$ These apparent discrepancies from our study may be due to differences in the TCM syndromes in the participants recruited and differences in treatment techniques.

In our study, it is interesting that conjunctival redness was reduced by AC. Previously it has been shown that conjunctival redness corresponds to the presence of inflammatory cells. ${ }^{18} \mathrm{~T}$ cells have been implicated in the pathogenesis of dry eye, and Th1, Th2 and Th17 cytokines have been associated with this condition. ${ }^{19} 20$ The normal human ocular surface expresses receptors for all major cytokines produced by Th lymphocytes, and propagation of the inflammatory response in dry eye depends on the downstream pathways activated by these receptors. ${ }^{21}$ Currently we found that AC reduced the levels of the Th1 cytokine TNF- $\alpha$ in the tear. Previously it was found that corticosteroid eye-drops reduced the level of TNF- $\alpha$ in the tear of postcataract surgery patients with dry eye. ${ }^{22}$ This suggests that tear TNF- $\alpha$ concentration is a measure of inflammation in dry eye that is responsive to treatment, and AC may act via this mechanism. The reduction in TNF- $\alpha$ was contributed in part by a few participants with rather high pretreatment levels of TNF- $\alpha$ (online supplementary efigure 5 ). From the data it was not possible to determine if these participants may be more responsive to the treatment due to severity of disease or if the phenomenon may be a regression to the mean. However, there may be alternative mechanisms for explaining an increase in conjunctival hyperaemia and reduction of TNF- $\alpha$ apart from immune mechanisms. For example, the mechanisms may involve neurological stimulation of tear fluid production that did not alter Schirmer's tests or nerve mechanisms that alter epithelial production of TNF- $\alpha$.

A previous study using AC showed that this procedure can increase the level of tear lactoferrin, at least transiently. ${ }^{23}$ Lactoferrin is an important multifunctional tear protein that has antimicrobial and anti-inflammatory effects and has been found to be reduced in the tear of patients with dry eye. ${ }^{24}$ It was unclear if patients with dry eye in that study ${ }^{23}$ had yin-deficiency.

A study in rabbits documented a change in tear proteins, for example, an increase in tear lipocalin after $\mathrm{AC}^{25}$ but tear cytokines were not evaluated. Another rabbit study showed morphological changes in the lacrimal gland after AC, but no mechanistic explanation had been offered. ${ }^{26}$

The fundamental principles in conventional medicine and TCM are vastly different. Conventional medicine employs a more reductionist approach while TCM uses a holistic approach. Despite the differences in approach, it is remarkable that AC, a TCM modality relieved dry eye symptoms through achieving improvement of yin (TCM objective) which somehow improved ocular pathology. There may be great heterogeneity in the actual aetiology of dry eye disease, even though we excluded patients with known systemic diseases. These interindividual differences are commonly encountered in idiopathic dry eye or hormone-related dry eye and may explain differing responses to the TCM treatment.

Our randomised study is the first to use objective, robust clinical end points as well as parameters relevant to the scientific basis of dry eye pathology. The standardisation of treatment protocols at one single centre for treatment is critical to elicit treatment effects. Moreover, 
all treatment are performed by nationally accredited, well-qualified Chinese medicine physicians. The low dropout rate is also a strength of the current trial.

We did not use separate control groups for AC and $\mathrm{HB}$, that is, sham $\mathrm{AC}$ and placebo drugs, respectively. Doing such a study would have doubled the numbers required for controls and would be non-feasible given the resources available and the existing complicated logistics involving two separate evaluation and treatment centres. Not having separate controls may introduce bias to the measurement of the subjective outcome since patients were not masked to having TCM treatment. However, we mitigate this with investigator masking and objective measurements. A previous multicentre randomised study in moderate to severe dry eye participants showed that AC reduced dry eye symptoms at the end of 8 weeks, to a greater extent than patients using only AT. ${ }^{27}$ Our study did not address long-term changes beyond 1 month.

We determined that the number of individuals with improvement in SPEED is different between AC and HB groups, but the mean final SPEED at 1 month is not different between groups. This is likely due to a few outlying participants in the HB and AT group who had more than 12 points of improvement (online supplementary efigure 3 top row, middle and right columns). This may also be related to individuals with worsening of SPEED in the AC group that induces a higher mean SPEED at 1 month. The observation of participants which had worsening of symptoms after AC indicate that there may be other unexamined factors that could help to predict response or otherwise in the AC group. Although the overall SPEED scores at baseline were not significantly different between AC and HB groups, there were a few participants who appeared to have higher baseline values in AC, which responded with a drop after treatment.

For the reasons discussed above, it would be erroneous to form conclusions purely based on symptoms. In particular, the proportion of the symptom responders may be influenced by lack of participant masking. The findings related to symptoms should be interpreted in the context of more objective tests. Another limitation is that we did not have re-evaluation after more prolonged cessation of treatment. A further evaluation of trial end points 4 weeks after discontinuation of the TCM treatment would be useful. The differential effects may persist if some fundamental processes have been altered or may abate, if they are related to transient processes.

Many other tear cytokines were not tested in the study. Hypotheses involving nerve sensitising agents such as bradykinin, serotonin, SP and (nerve growth factor) NGF; nerve function studies, systemic changes in mood and pain perception were not evaluated. Type I errors may be at play given the number of cytokines measured. Although Singapore weather is equatorial and fairly constant throughout the year compared with temperate climates, we cannot exclude the possibility that patients could be affected by different environmental conditions from March to December in the year 2015.
We acknowledge that dry eye is a complex condition and although we excluded known systemic diseases, we did not evaluate mental state, corneal nerve function or intensity of using computers. Because the mechanism of $\mathrm{AC}$ is not known in relation to these parameters, there may be unknown confounders that can explain the current observations.

Current treatment of dry eye involves predominantly eye-drops which usually provides only temporary symptomatic relief. Patients find continued treatment to be ineffective, costly or troublesome. Moreover, the current study suggests that $30 \%$ of people with dry eye may not find relief from ATs. As such, alternative treatments should be explored. ${ }^{2}$ For patients with mild to moderate dry eye who wish to have additional treatment, AC can be considered, provided that there is access to suitably trained, preferably nationally accreditated professionals. As AC is performed by practitioners, it may result in higher patient compliance compared with self-administration of lubricants.

Our previous study found that a majority of TCM practitioners believe TCM to be effective in dry eye. Seventy-four per cent has had experience managing dry eye. ${ }^{28}$ However, it is unclear if TCM is cost-effective. It would be useful to survey patients who have received TCM treatment regarding its perceived cost and efficacy.

Further studies should delineate the exact role of TCM treatment in the entire spectrum of dry eye disease, as more severe disease is currently treated with anti-inflammatory agents, and it is not certain if $\mathrm{AC}$ adds to the benefit conferred by anti-inflammatory agents. ${ }^{2}$

AC treatment in adult patients with mild to moderate dry eye is associated with demonstrable molecular evidence of reduced inflammation. It is unknown if these results are generalisable to the general population or other populations. AC and the evaluated $\mathrm{HB}$ formulation were safe up to a month.

Acknowledgements Xavier Le Geuzennec from Curiox Pte helped to analyse the cytokines.

Contributors LT conceived the study and wrote the initial manuscript, PL performed TCM treatment, with supervision from Q-PW, AHH analysed the cytokines, RUA, J-HT contributed to thermographic analysis and H-MH contributed to statistical analysis. All authors critically revised the paper for important intellectual content and approved the final version to be published. LT had full access to all of the data in the study and takes responsibility for the integrity of the data and the accuracy of the data analysis. LT and PL supervised the study and are the guarantors.

Funding This research is funded by Singapore Ministry of Health's Traditional Chinese Medicine Clinical Research Grant (TCMCRG 3103001). This research is also supported by the Singapore National Research Foundation under its clinician scientist award NMRC/CSA/045/2012 and NMRC/CSA-SI/0017/2017, administered by the Singapore Ministry of Health's National Medical Research Council.

Competing interests LT is supported by an award NMRC/CSA/045/2012 and NMRC/CSA-SI/0017/2017 from National Medical Research Council, Singapore.

Patient consent Not required.

Ethics approval The study was approved by the central Institutional Review Board of Singapore Health services, adhering to the tenets of the Declaration of Helsinki for human research.

Provenance and peer review Not commissioned; externally peer reviewed. 
Data sharing statement No additional data are available.

Open access This is an open access article distributed in accordance with the Creative Commons Attribution Non Commercial (CC BY-NC 4.0) license, which permits others to distribute, remix, adapt, build upon this work non-commercially, and license their derivative works on different terms, provided the original work is properly cited and the use is non-commercial. See: http://creativecommons.org/ licenses/by-nc/4.0/

(C) Article author(s) (or their employer(s) unless otherwise stated in the text of the article) 2018. All rights reserved. No commercial use is permitted unless otherwise expressly granted.

\section{REFERENCES}

1. Tong L, Tan J, Thumboo J, et al. Dry eye. BMJ 2012;345:e7533.

2. Craig JP, Nichols KK, Akpek EK, et al. TFOS DEWS II Definition and Classification Report. Ocul Surf 2017:15:276-83.

3. Yang L, Yang Z, Yu H, et al. Acupuncture therapy is more effective than artificial tears for dry eye syndrome: evidence based on a meta-analysis. Evid Based Complement Alternat Med 2015;2015:1-11

4. Lim P, Lan WW, Wei PQ, et al. Traditional Chinese medicine in dry eye disease. In: Eye diseases and disorders: a complete guide: iConcept Press, 2014

5. Chang YH, Lin HJ, Li WC. Clinical evaluation of the traditional chinese prescription Chi-Ju-Di-Huang-Wan for dry eye. Phytother Res 2005;19:349-54.

6. Grönlund MA, Stenevi U, Lundeberg T. Acupuncture treatment in patients with keratoconjunctivitis sicca: a pilot study. Acta Ophthalmol Scand 2004;82(3 Pt 1):283-90.

7. Shin MS, Kim JI, Lee MS, et al. Acupuncture for treating dry eye: a randomized placebo-controlled trial. Acta Ophthalmol 2010;88:e328-33.

8. Nepp J, Wedrich A, Akramian J, et al. Dry eye treatment with acupuncture. A prospective, randomized, double-masked study. Adv Exp Med Biol 1998;438:1011-6.

9. Tseng KL, Liu HJ, Tso KY, et al. A clinical study of acupuncture and SSP (silver spike point) electro-therapy for dry eye syndrome. Am J Chin Med 2006:34:197-206.

10. Lee MS, Shin BC, Choi TY, et al. Acupuncture for treating dry eye: a systematic review. Acta Ophthalmol 2011;89:101-6.

11. Wei Q-P, Rosenfarb A, L-n L. Ophthalmology in Chinese medicine: People's Medical Publishing House Co, LTD, 2011:345.

12. Lin T, Gong L, Liu X, et al. Fourier-domain optical coherence tomography for monitoring the lower tear meniscus in dry eye after acupuncture treatment. Evid Based Complement Alternat Med 2015;2015:1-10.
13. Tong L, Beuerman R, Simonyi S, et al. Effects of punctal occlusion on clinical signs and symptoms and on tear cytokine levels in patients with dry eye. Ocul Surf 2016;14:233-41.

14. Laupacis A, Sackett DL, Roberts RS. An assessment of clinically useful measures of the consequences of treatment. $N$ Engl $J$ Med 1988;318:1728-33.

15. Yeo S, Tan JH, Acharya UR, et al. Longitudinal changes in tear evaporation rates after eyelid warming therapies in meibomian gland dysfunction. Invest Ophthalmol Vis Sci 2016;57:1974-81.

16. Nepp J, Derbolav A, Haslinger-Akramian J, et al. [Effect of acupuncture in keratoconjunctivitis sicca]. Klin Monbl Augenheilkd 1999;215:228-32.

17. Lan W, Tong L. Acupuncture has effect on increasing tear break-up time: acupuncture for treating dry eye, a randomized placebocontrolled trial. Acta Ophthalmol 2012;90:e73.

18. Ferrari G, Rabiolo A, Bignami F, et al. Quantifying ocular surface inflammation and correlating it with inflammatory cell infiltration in vivo: a novel method. Invest Ophthalmol Vis Sci 2015;56:7067.

19. El Annan J, Chauhan SK, Ecoiffier T, et al. Characterization of effector T cells in dry eye disease. Invest Ophthalmol Vis Sci 2009:50:3802-7.

20. Meadows JF, Dionne K, Nichols KK. Differential profiling of T-Cell cytokines as measured by protein microarray across dry eye subgroups. Cornea 2016;35:329-35.

21. Pflugfelder SC, Corrales RM, de Paiva CS. T helper cytokines in dry eye disease. Exp Eye Res 2013;117:118-25.

22. Jee D, Park M, Lee HJ, et al. Comparison of treatment with preservative-free versus preserved sodium hyaluronate $0.1 \%$ and fluorometholone $0.1 \%$ eyedrops after cataract surgery in patients with preexisting dry-eye syndrome. J Cataract Refract Surg 2015;41:756-63.

23. Shi JL, Miao WH. [Effects of acupuncture on lactoferrin content in tears and tear secretion in patients suffering from dry eyes: a randomized controlled trial]. Zhong Xi Yi Jie He Xue Bao 2012;10:1003-8

24. Rageh $\mathrm{AA}$, Ferrington DA, Roehrich $\mathrm{H}$, et al. Lactoferrin expression in human and murine ocular tissue. Curr Eye Res 2016;41:883-9.

25. Qiu X, Gong L, Sun X, et al. Efficacy of acupuncture and identification of tear protein expression changes using iTRAQ quantitative proteomics in rabbits. Curr Eye Res 2011;36:886-94.

26. Gong L, Sun X. Treatment of intractable dry eyes: tear secretion increase and morphological changes of the lacrimal gland of rabbit after acupuncture. Acupunct Electrother Res 2007;32:223-33.

27. Kim TH, Kang JW, Kim KH, et al. Acupuncture for the treatment of dry eye: a multicenter randomised controlled trial with active comparison intervention (artificial teardrops). PLoS One 2012; 7:e36638.

28. Lan W, Lee SY, Lee MX, et al. Knowledge, attitude, and practice of dry eye treatment by institutional Chinese physicians in Singapore. ScientificWorldJournal 2012;2012:1-9. 